Check for updates

Cite this: RSC Adv., 2017, 7, 49330

Received 15th August 2017 Accepted 9th October 2017

DOI: 10.1039/c7ra09011k

rsc.li/rsc-advances

\title{
A novel dual-emission QDs/PCDs assembled composite nanoparticle for high sensitive visual detection of $\mathrm{Hg}^{2+} \uparrow$
}

\author{
Jian Cui, ${ }^{a}$ Tianjiao Liu, ${ }^{a}$ Dapeng Liang, ${ }^{a}$ Jiale Liu, ${ }^{\mathrm{b}}$ Kai Zhang, (D) ${ }^{\mathrm{b}}$ Bai Yang (D) ${ }^{\mathrm{b}}$ \\ and Yang Ning (iD *ab
}

Traditional fluorescent probe detection is an established technique for enhancing or quenching luminescence intensity; however, a color changeable fluorescent material is still a challenge in many target detection fields. Herein, a dual-emission quantum dots (QDs)/polymer carbon dots (PCDs) assembled composite nanoparticle was prepared via a simple self-assembly method. By choosing the QDs and PCDs source, the luminescence color of the composite nanoparticles can be controlled all over the visible light spectrum. The composite material possesses high sensitivity and selectivity with $\mathrm{Hg}^{2+}$; with increasing concentration of $\mathrm{Hg}^{2+}$, the luminescence color of the composite material changes from pink to violet, and at last blue. The detection limit of $\mathrm{Hg}^{2+}$ was $10 \mathrm{nM}$ by luminescence spectrum, and $100 \mathrm{nM}$ by visual observation, so it may be utilized for visual detection in environmental science and biology.

\section{Introduction}

Composite nanomaterials are very promising materials in photocatalytic, ${ }^{1,2}$ bio-imaging, ${ }^{3,4}$ environmental detecting, ${ }^{5}$ therapy, ${ }^{6}$ and other fields due to their excellent optical, electrical, magnetic, and thermal properties. ${ }^{7}$ With the development of this field, controllable structure, sensitive responsiveness, and low toxicity have become the main focus of many researchers. ${ }^{8}$ The controllable structure may adjust the physical and chemical properties of materials according to different applications, and sensitive responsiveness could exhibit exciting functions of the material. Further, low toxicity will enable the application fields of these materials to be widened.

Semiconductor nanocrystals are one kind of classic fluorescent quantum dots (QDs) which have been researched in recent decades among many areas including chemistry, ${ }^{9}$ biology, ${ }^{10}$ medicine, ${ }^{11}$ pharmacology, ${ }^{6}$ and videography ${ }^{12}$ because of its fascinating optical properties. Usually, luminescence of semiconductor nanocrystals can be altered among the whole visible light spectrum according to the quantum size effect. However, the inherent deleterious crisis from the heavy metal ions of QDs

\footnotetext{
${ }^{a}$ Key Lab of Groundwater Resources and Environment Ministry of Education, College of Environment and Resources, Jilin University, 2699 Qianjin Avenue, Changchun, 130012, P. R. China. E-mail: ningyang@jlu.edu.cn

${ }^{b}$ State Key Laboratory of Supramolecular Structure and Materials, College of Chemistry, Jilin University, Changchun, 130012, P. R. China

$\dagger$ Electronic supplementary information (ESI) available. See DOI: 10.1039/c7ra09011k
}

makes people hesitate with applications of these semiconductor nanocrystals. ${ }^{4,13-15}$ Meanwhile, carbon dots (CDs), especially polymer carbon dots (PCDs) which are considered as a promising material, have been developed rapidly in the last few years. ${ }^{2,16}$ Because the main constituent of PCDs is carbon, which possesses very low toxicity, ${ }^{17}$ they are considered as an environmentally friendly nanomaterial. Therefore, many researches on the synthesis and application of PCDs have been explored such as water ion detection, ${ }^{18}$ trace elements analysis, ${ }^{19}$ an organic small molecular probe, ${ }^{20}$ photo catalyst,${ }^{21}$ and the environmental energy field. ${ }^{22}$ The adjustment of PCDs comes from its excitation-dependent property; thus, tunable luminescence of PCDs with high fluorescent intensity in a wider visible region is still a challenge.

Here, we fabricated a dual-emission QDs/PCDs assembled composite nanoparticle by a simple self-assembly process. Luminescence of the dual-emission composite nanoparticle could be adjusted in a wider range to control the fluorescent QDs and the ratio between the QDs and the PCDs. Owing to a decrease of the heavy metal content in the composite material and the inter binding of the nanoparticles, toxicity from the QDs in the composite material would be reduced greatly. We also found that this dual-emission composite nanomaterial possessed an excellent property of $\mathrm{Hg}^{2+}$ ion detection; the detection limit of $\mathrm{Hg}^{2+}$ was $10 \mathrm{nM}$ by luminescence spectrum, and $100 \mathrm{nM}$ by visual observation. ${ }^{23}$ Because the PCDs were rich in amino groups and carboxyl groups, they could be modified easily and be used in further applications such as special recognition, biological probes, tissue imaging, etc. 


\section{Experimental section}

Materials

Tellurium powder (99.8\%), poly(maleic anhydride-alt-1-octadecene) (PMAO) powder, chloromethylstyrene $N, N$-dimethyloctadecylamine, and 4-vinylbenzyl chloride were purchased from Aldrich, USA. Sodium borohydride $\left(\mathrm{NaBH}_{4}, 99 \%\right)$ and cadmium chloride $\left(\mathrm{CdCl}_{2} \cdot 2.5 \mathrm{H}_{2} \mathrm{O}, 99 \%\right)$ were purchased from Sinopharm Chemical Reagent Co., Ltd., China, N,N-dimethyloctadecylamine and mercaptopropionic acid (MPA) were purchased from Acros, USA. Ethylenediamine (EDA) was purchased from Aladdin Company, China. Acetone, chloroform, and ethanol were all AR grade from Beijing Chemical Works, China. All materials were used as received.

\section{Characterization}

High-resolution transmission electron microscope photos (HRTEM) were recorded on a JEM-2100F and an FEI Tecnai F20. The composite samples' corresponding TEM-EDX elemental mapping data were measured using energy-dispersive X-ray. The FT-IR spectra were recorded in the range of 4000$450 \mathrm{~cm}^{-1}$ using a Bruker Vector 22 spectrophotometer. Laser confocal scanning microscope (LCSM) images were obtained on a FV 1000 from OLYMPUS. Ultraviolet photoelectron spectrometer (UPS) data was measured using PREVAC UPS equipment. Fluorescence lifetime (FLT) was performed on Edinburgh FLS980 equipment, with a $100 \mathrm{~Hz}$ xenon lamp as light source. UV spectra were recorded with an ultraviolet and visible spectrophotometer (UV), Lambda_800 America and a fluorescence emission spectrum (FL) was recorded using SHIMADZU 5301PC equipment, with a $150 \mathrm{~W}$ xenon lamp and emission located in the 330-420 nm range. All photos were collected using a digital camera, and the fluorescent photos were collected using an ultraviolet laser (365 $\mathrm{nm}$ excitation).

\section{Synthesis of PCDs}

The amphiphilic PCDs were synthesized by a hydrothermal method. An aqueous solution with $500 \mu \mathrm{L}$ EDA and $150 \mathrm{mg}$ PMAO in a polytetrafluorethylene (PTFE) reaction container was heated at $200{ }^{\circ} \mathrm{C}$ for 6 hours in a muffle furnace. After cooling to room temperature, the product was dialyzed with deionized water in a dialysis bag $\left(M_{\mathrm{w}}=8000\right)$. A tiny amount of yellow solid was removed by a filter. The as prepared solution was stored for following experiments.

\section{Preparation of hydrophobic cadmium telluride (CdTe) QDs}

The surfactant octadecyl-p-vinyl-benzyldimethylammonium chloride (OVDAC) and the CdTe QDs were synthesized according to our previous paper. ${ }^{24}$ The original concentration of $\mathrm{Cd}^{2+}$ was $10^{-3} \mathrm{M}$, and the molar ratio of $\mathrm{Cd}^{2+} / \mathrm{MPA} / \mathrm{HTe}^{-}$was fixed at $1: 2.4: 0.2$. To alter the surface polarity of the NPs, $30 \mathrm{~mL}$ CdTe aqueous solution and $6 \mathrm{~mL}$ OVDAC chloroform solution $(5 \mathrm{mg}$ $\mathrm{mL}^{-1}$ ) were mixed under vigorous stirring for $15 \mathrm{~min}$, then the CdTe QDs product was transferred to the chloroform phase.
Fabrication of dual-emission QDs/PCDs assembled composite nanoparticles

A $20 \mathrm{~mL}$ aqueous solution with $1.5 \mathrm{~mL}$ PCDs aqueous solution and $500 \mu \mathrm{L}$ CdTe QDs chloroform solution was kept ultrasonicating for $15 \mathrm{~min}$. When the cloudy solution turned to clear again, the dual-emission QDs/PCDs assembled composite nanoparticles were obtained.

\section{Detection of $\mathrm{Hg}^{2+}$}

Typically, $2 \mathrm{~mL}$ of dual-emission composite nanoparticle aqueous solution prepared above was added into a cuvette. Certain amounts of $\mathrm{Hg}^{2+}$ and deionized water were added into the cuvette to make the total volume be $10 \mathrm{~mL}$, and the final concentrations of $\mathrm{Hg}^{2+}$ were 1, 5, 10, 20, 50, 100, 200, 300, 400, and $500 \mathrm{nM}$. The fluorescence spectra of the dual-emission probe under a single wavelength excitation at $360 \mathrm{~nm}$ were recorded by a fluorescence spectrophotometer. All sensitivity and selectivity measurements were conducted in triplicate. The visible ion response was observed under a $365 \mathrm{~nm}$ UV lamp. To test for selectivity of $\mathrm{Hg}^{2+}$ among other metal ions, different kinds of metal ions including $\mathrm{K}^{+}, \mathrm{Pb}^{2+}, \mathrm{Co}^{2+}, \mathrm{Ce}^{3+}, \mathrm{Sr}^{2+}, \mathrm{Al}^{3+}$, and $\mathrm{Cr}^{3+}$ were added to the mixture solution in the same way to make their concentrations be $500 \mathrm{nM}$.

\section{Results \& discussion}

The structure and assembly process of dual-emission assembled composite nanoparticles are illustrated in Fig. 1. A two-step assembly method was applied to obtain the dual-emission assembled composite nanoparticles. First, PCDs with alkyl chains on the surface and CdTe QDs modified with hydrophobic surfactant were prepared separately. The PCDs were synthesized by a hydrothermal condensation reaction between PMAO and EDA which were rich with maleic units and amine units. The PMAO is a kind of alternating copolymer, and it has both the hydrophilic group-maleic anhydride and hydrophobic group - long carbon chain. The alkyl chains from 1-octadecene in PMAO were kept during the hydrothermal reaction, so amphiphilic PCDs were obtained. Since there were many negative charges on the surface of CdTe QDs, the aqueous CdTe QDs could be assembled easily with positively charged OVDAC

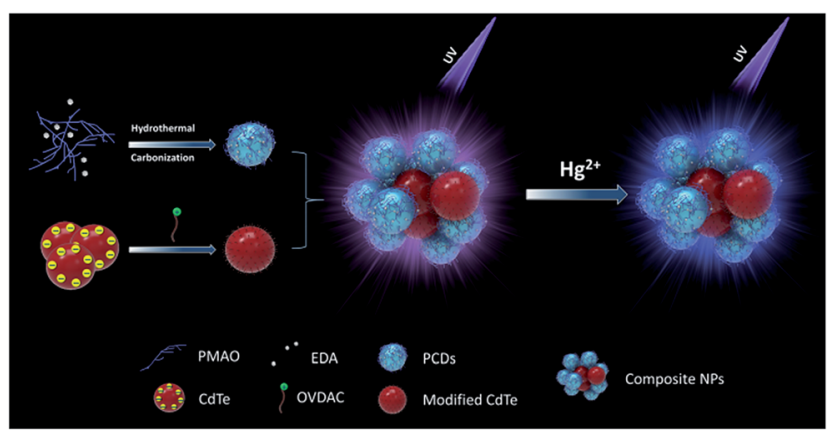

Fig. 1 Schematic illustration of the formation of dual-emission fluorescent composite nanoparticles and the detection of $\mathrm{Hg}^{2+}$. 
by electrostatic interaction. The CdTe QDs modified with hydrophobic surfactant were transferred into the chloroform phase. Then the dual-emission assembled composite nanoparticles were obtained by assembly of amphiphilic PCDs and hydrophobic CdTe QDs under ultrasonic conditions because of the hydrophobic-hydrophobic interaction. During this process, two different luminescent properties of the PCDs and QDs were kept well in the composite nanoparticles.

The structure of PCDs was investigated by FT-IR spectroscopy (Fig. 3a). The sharp peaks of PCDs at $2923 \mathrm{~cm}^{-1}$ and $2852 \mathrm{~cm}^{-1}$ were similar to PMAO, which were attributed to the stretching vibrations of $-\mathrm{CH}_{3}$ and $\mathrm{CH}_{2}$. This meant the long alkyl chains from the PMAO were kept during the formation of PCDs under the hydrothermal condition, and it could combine with the CdTe QDs by hydrophobic-hydrophobic interaction. Compared with PMAO, there were three more obvious peaks at $1411 \mathrm{~cm}^{-1}, 1570 \mathrm{~cm}^{-1}$, and $3396 \mathrm{~cm}^{-1}$ in the PCDs. The peaks at $1411 \mathrm{~cm}^{-1}$ and $1570 \mathrm{~cm}^{-1}$ were attributed to the $\mathrm{N}-\mathrm{H}$ stretching vibration, and the peak at $3396 \mathrm{~cm}^{-1}$ was because of the stretching vibration of $-\mathrm{NH}_{2}$ groups and $-\mathrm{OH}$ groups. The FT-IR studies confirmed that the PCDs were composed of PMAO and EDA according to the hydrothermal reaction, and the important functional groups were kept separately. The fluorescence spectra are shown in Fig. 2b. The PCDs possessed an excitation-dependent property the same as many kinds of CDs in the literature ${ }^{25,26}$ and the optimal emission peak of the PCDs was at $433 \mathrm{~nm}(\mathrm{ex}=365)$, which was bright blue under UV light. The morphology of PCDs was observed by TEM as shown in Fig. 2c. The PCDs were nearly spherical and well-dispersed without any aggregation. Their size distribution (Fig. 2c insert) indicated that their average size was about $2.8 \mathrm{~nm}$ according to the statistics. Their interplanar distances were
$0.21 \mathrm{~nm}$ and $0.32 \mathrm{~nm}$ which is consistent with the (100) and (002) crystallographic facets of graphite carbon. ${ }^{27}$

In order to obtain a varied dual-emission assembled composite material, red emission CdTe QDs were chosen for this preparation. The CdTe QDs aqueous dispersion was synthesized according to our previous paper. ${ }^{24}$ The absorption and fluorescence spectra of CdTe QDs (Fig. 3a) showed that there was an obvious absorption in the range of $610 \mathrm{~nm}$, and the emission wavelength of the CdTe QDs was about $640 \mathrm{~nm}$. As shown in the TEM image (Fig. 3b), the sizes of the QDs were uniform, and their average diameter was about $3.2 \mathrm{~nm}$. Their interplanar distances were $0.23 \mathrm{~nm}$ which is consistent with the (220) crystallographic facet of cubic CdTe. ${ }^{28}$

Then, the hydrophilic QDs were transferred to chloroform with OVDAC, which is a surfactant with alkyl chains. A red transparent chloroform dispersion was gained meaning the CdTe QDs were modified successfully by hydrophobic chains without any aggregation.

After the self-assembly process, dual-emission composite nanoparticles were obtained. In the fluorescence spectra (Fig. 4a), two kinds of luminescence from PCDs and QDs had been kept well in the dual-emission composite nanoparticles, and the composite light is about orange which was dependent on the ratio between the amounts of the two nanoparticles. The TEM and HRTEM images show that the composite nanoparticles are composed of two kinds of nanoparticles; two kinds of interplanar distances could be observed which correspond to the PCDs and QDs.

In order to prove that two kinds of nanoparticles were indeed assembled in one composite nanoparticle, element mapping TEM images were taken to confirm distribution of the particles. In Fig. $\mathrm{S} 1, \uparrow$ the element mappings of $\mathrm{C}, \mathrm{Cd}$, and Te were taken

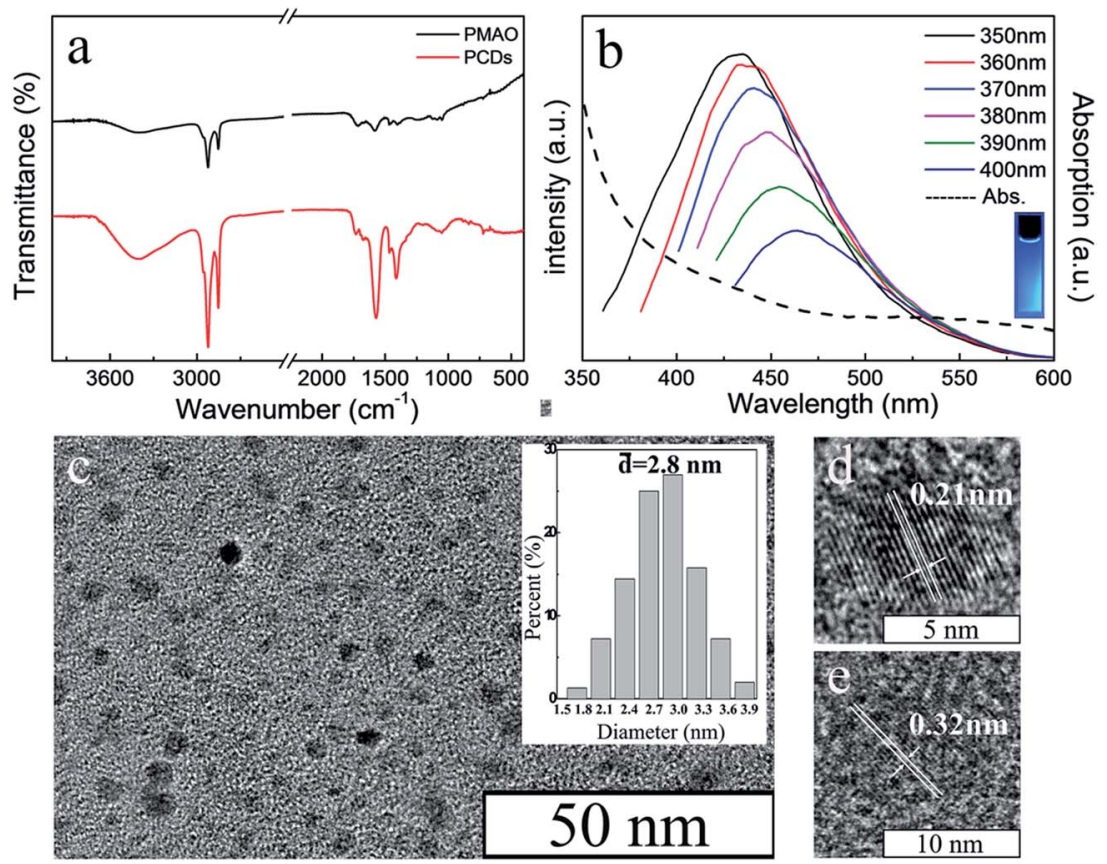

Fig. 2 (a) FT-IR spectra of PMAO and PCDs. (b) Photoluminescence and absorption spectrum of PCDs. (c) TEM image and the size distribution (insert) of PCDs. (d) (e) HRTEM images of PCDs. 

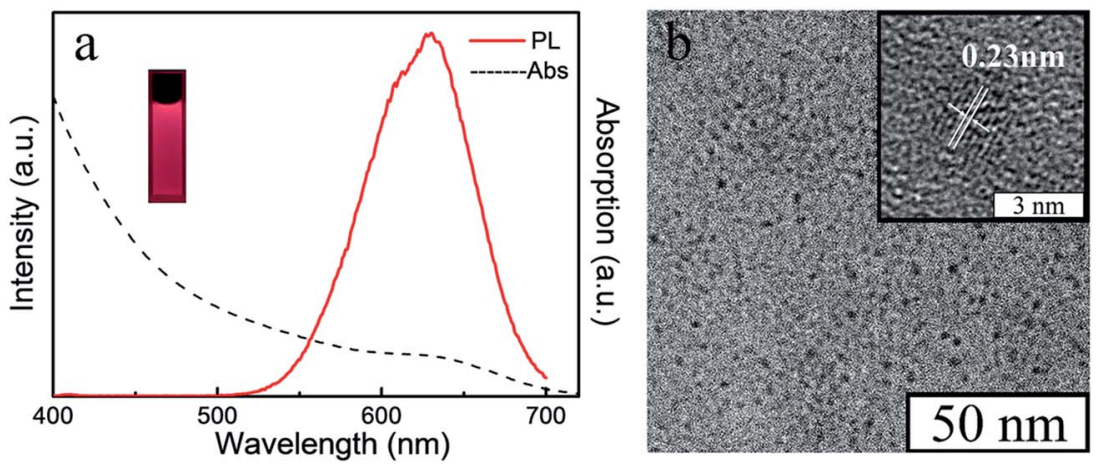

Fig. 3 The UV absorption and photoluminescence spectrum (a), the fluorescent photo under the UV light (insert), the TEM image (b) and the HRTEM image (insert) of aqueous CdTe QDs.
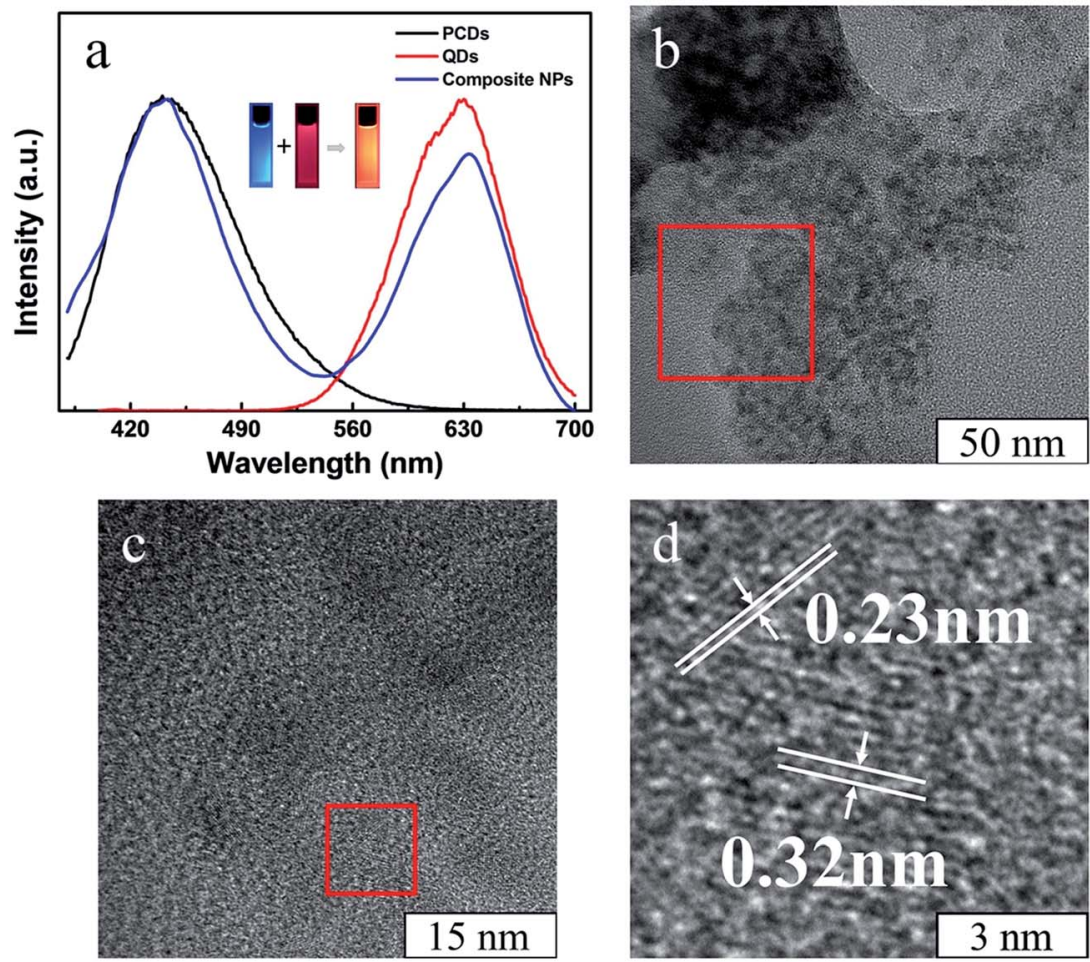

Fig. 4 (a) The normalized photoluminescence spectrum of PCDs, CdTe QDs, and the dual-emission fluorescent composite nanoparticles (ex = $370 \mathrm{~nm}$ ). (b) TEM images of the composite nanoparticles. (c) HRTEM image of the red area in (b). (d) HRTEM image of the red area in (c).

in the same area. Although the density of the elements was very low, it still could be found that elements Cd and Te were in about the same area, which was inside the composite nanoparticle. At the edge of the composite nanoparticles, there should be some CDs, so that CdTe QDs with alkyl chains could be stable in the aqueous solution. Carbon mapping could not be observed clearly because a thin carbon film was on the copper grid which was used for TEM measurements and thus caused a lot of interference. Moreover, a confocal laser scanning microscope was used to investigate the fluorescent property of a single composite nanoparticle (Fig. S2 $\dagger$ ). Although the luminescence of smaller composite nanoparticles was not bright enough to be recorded, the confocal laser scanning microscope image still showed that the larger nanoparticles exhibited different fluorescent colors under different emission conditions which were accordant with the fluorescent spectra.

The fluorescent spectra of the composite nanoparticles were somewhat different from the spectra of single nanoparticles under the same concentration and measurement conditions in Fig. 4a. There was a slight red shift of the fluorescence peak of the QDs during formation of the composite nanoparticles. This may be due to aggregation of the CdTe QDs. Moreover, the composite nanoparticles assembled by CdTe QDs and the PMAO were prepared for comparison (Fig. S3 $†$ ). Under the same conditions, the fluorescence intensity of the CdTe in the dualemission composite nanoparticles was much higher than in 
the CdTe/PMAO composite nanoparticles; it was considered that the obviously fluorescence intensity change would be due to fluorescence resonance energy transfer (FRET) from the PCDs. In the HRTEM of the composite nanoparticles (Fig. 4d), the distance between the PCDs and the QDs was far less than $10 \mathrm{~nm}$, and the emission wavelength range of PCDs about $433 \mathrm{~nm}$ was just in the absorption wavelength range of QDs. So, the composite nanoparticles possessed basic conditions for the FRET. Then, the fluorescence lifetime of the PCDs (Fig. 5a) was used to investigate the luminescent process of the composite nanoparticles. Compared with single PCDs, the fluorescence lifetime of PCDs in the composite nanoparticles reduced from $5.1 \mathrm{~nm}$ to $4.8 \mathrm{~nm}$; this meant part of the energy would transfer from PCDs to the QDs. The ultraviolet photoelectron spectrometer data (Fig. 5b) indicated that the band gap of the PCDs was about $3.4 \mathrm{eV}$; this was much wider than $1.88 \mathrm{eV}$ which was the band gap of the QDs. ${ }^{29}$ So, the energy from PCDs was enough to excite the QDs. Therefore, the luminescence addition of QDs was due to the FRET from PCDs. ${ }^{30}$

More colorful composite materials could be fabricated by different kinds of CdTe QDs and varying the ratio between the PCDs and QDs. As shown in the fluorescence spectra (Fig. 6a), the green, yellow, and red luminescent CdTe QDs, in which emission wavelength were $549 \mathrm{~nm}, 565 \mathrm{~nm}$, and $640 \mathrm{~nm}$ respectively, were used to prepare the composite nanoparticles. And three corresponding different color composite nanoparticles were obtained (Fig. 6b).

\section{Specific detection for heavy metal $\mathbf{H g}^{2+}$}

Both PCDs and QDs have been already successfully applied in ion detection by fluorescence intensity change. However,
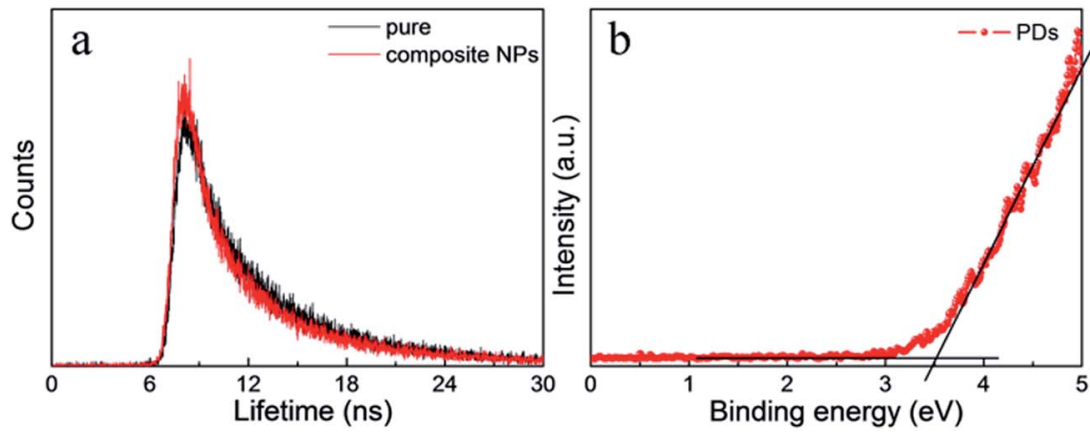

Fig. 5 (a) The fluorescence lifetime decay curve at $433 \mathrm{~nm}$ of PCDs and the PCDs in the composite nanoparticles. (b) UPS curve of the PCDs.
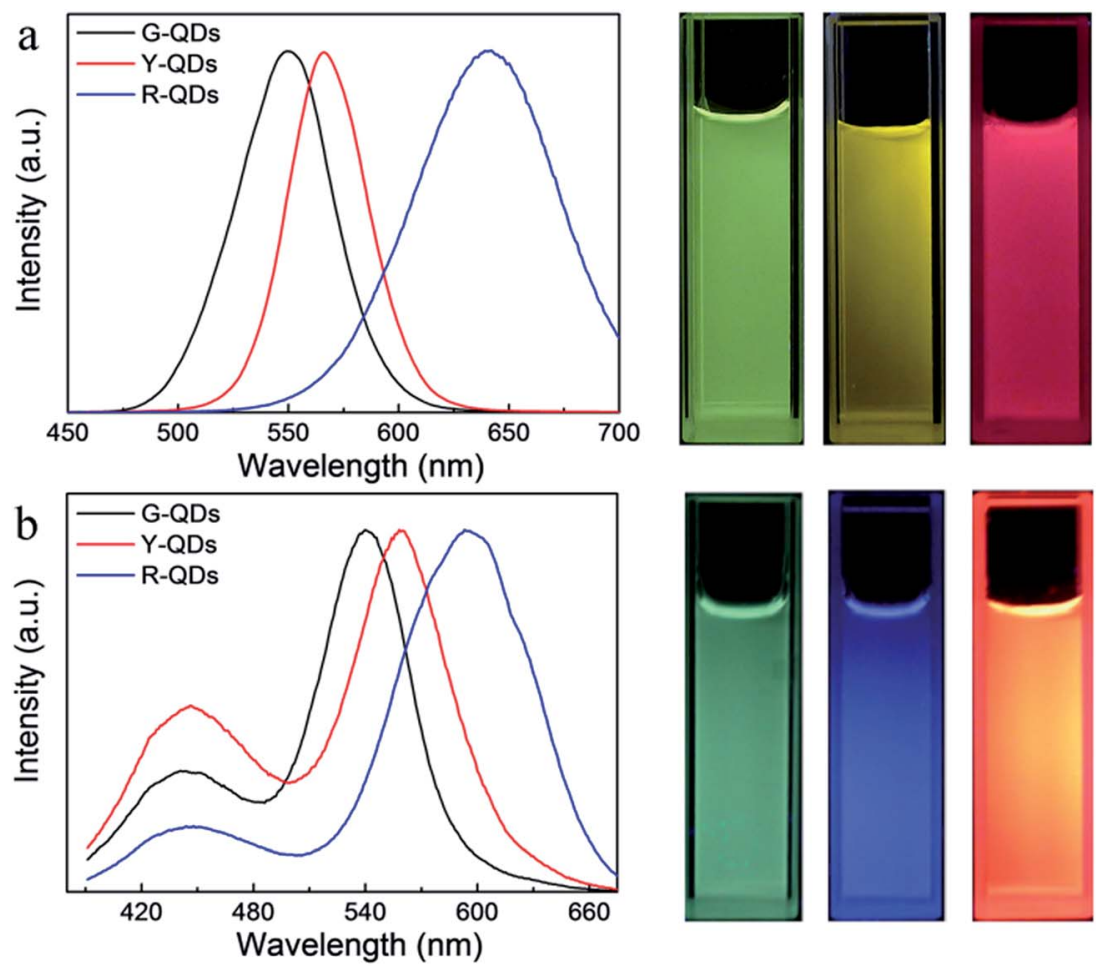

Fig. 6 Photoluminescence spectrum (a) and the photo under a UV light (insert) of green, yellow, and red QDs. Photoluminescence spectrum (b) and the photo under a UV light (insert) of corresponding composite nanoparticles (the luminescence intensities have been normalized). 

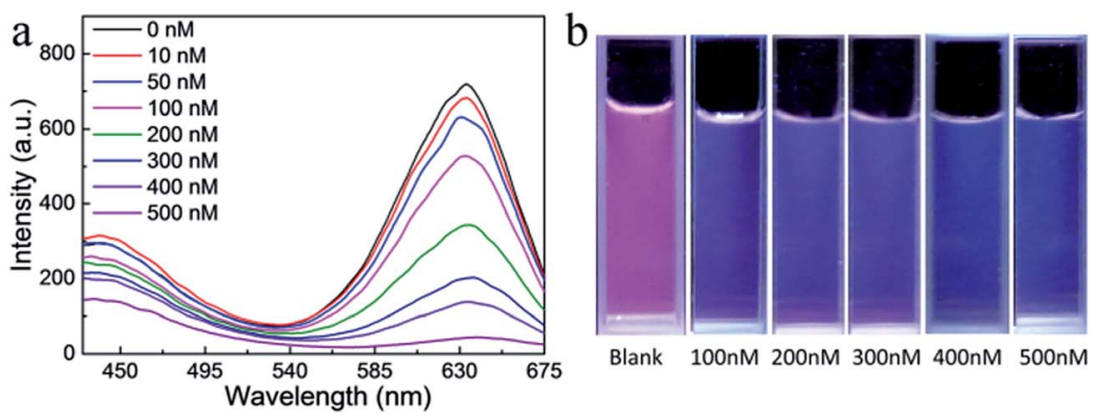

Fig. 7 (a) Photoluminescence spectrum of assembled nanoparticles with different concentrations of Hg ${ }^{2+}$. (b) Fluorescent digital images of assembled nanoparticles aqueous solution with different concentrations of $\mathrm{Hg}^{2+}$.

a fluorescence color changing detection system would be more sensitive in ion detection, especially for visual observation. The dual-emission assembled composite nanoparticles were excellent probes for detecting $\mathrm{Hg}^{2+}$. In fluorescence emission spectra (Fig. 7a), the fluorescence intensity of QDs and PCDs decreased with different ratios by adding different amounts of $\mathrm{Hg}^{2+}$. Because the composite color fluorescence of the dual-emission assembled composite nanoparticles were composed of blue from PCDs and red from QDs, therefore, the composite fluorescence would exhibit an observable color change from pink to cyan and finally to blue with different concentrations of $\mathrm{Hg}^{2+}$ (Fig. 7b). The detection limit for $\mathrm{Hg}^{2+}$ was $10 \mathrm{nM}$ by fluorescence spectrum, and $100 \mathrm{nM}$ by visual observation.

A sensitive detection for the dual-emission composite nanoparticles was composed of two mechanisms of the PCDs and CdTe QDs. For the PCDs, due to abundant carboxyl groups on the surface of PCDs, it was easy for them to combine with the positive charged $\mathrm{Hg}^{2+}$ ions, so quenching occurred. For the QDs, the binding constant between $\mathrm{Hg}^{2+}$ and Te was larger than the constant between $\mathrm{Cd}^{2+}$ and the $\mathrm{Te}$, so $\mathrm{Hg}^{2+}$ would replace $\mathrm{Cd}^{2+}$ on the surface of the QDs connecting with Te, which lead to quenching. With increasing the amounts of $\mathrm{Hg}^{2+}$, CdHgTe QDs would be formed, and a red shift would be observed in the fluorescent spectra. ${ }^{31}$ Because of the quenching of PCDs, the reduction of the FRET of the PCDs would lead to the weakness of QDs fluorescent intensity. Finally, the fluorescence intensity of PCDs and QDs reduced in the presence of $\mathrm{Hg}^{2+}$, and the reduction of QDs were more obvious. According to the FRET, the altering of the red emission intensity with $\mathrm{Hg}^{2+}$ in the dualemission composite nanoparticles system was rather greater than in the single QDs system, so the detection of $\mathrm{Hg}^{2+}$ by dualemission composite nanoparticles was more sensitive, and it could be used for visual detection under a low concentration of $\mathrm{Hg}^{2+}$. A comparison of the responses between the single PCDs or CdTe QDs and the composite nanoparticles had been studied as shown in Fig. S4. $\dagger$ The detection fluorescence intensity alteration of composite nanoparticles was much greater than with single nanoparticles. This indicated that the composite structure was established, and the FRET existed in the dual-emission assembled composite nanoparticles system. This special fluorescence property made the composite nanoparticles an ideal material for visual detection of $\mathrm{Hg}^{2+}$ ions. The response of the dual-emission fluorescent composite nanoparticles for $\mathrm{Hg}^{2+}$ detecting was very rapid. Under different concentration conditions, the fluorescence intensities of the composite nanoparticles could reach steady state within five minutes, thence it would have more advantages in practical applications.

To evaluate detection selectivity of the dual-emission fluorescent composite nanoparticles, different kinds of ions were used to investigate the fluorescent spectra (Fig. 8a) at a concentration of $500 \mathrm{nM}$. The fluorescence intensities of the composite nanoparticles with different ions had changed more or less. So, the fluorescence alteration intensity ratio $\left(\Delta I_{633}\right)$ $\Delta I_{433}$ ) was used to study the composite luminescence of
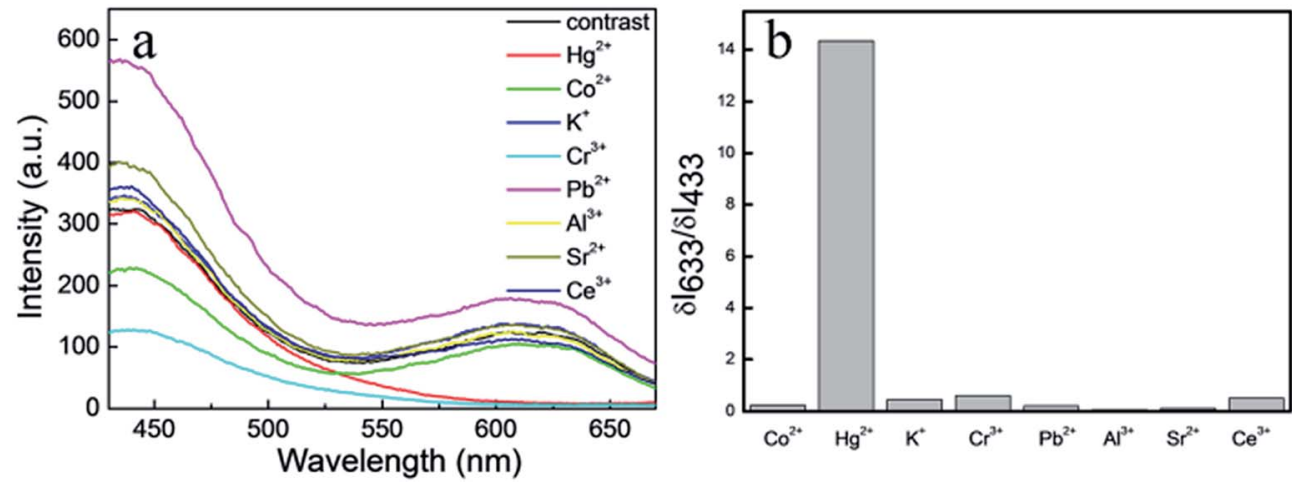

Fig. 8 (a) Photoluminescence spectrum of composite NPs with different metal ions where the concentration was $500 \mathrm{nM}$. (b) The $\Delta /_{633} / \Delta I_{433}$ values of different ions. 
assembly nanoparticles. With different metal ions including $\mathrm{Hg}^{2+}, \mathrm{Co}^{2+}, \mathrm{K}^{+}, \mathrm{Cr}^{3+}, \mathrm{Pb}^{2+}, \mathrm{Al}^{3+}, \mathrm{Sr}^{2+}$, and $\mathrm{Ce}^{3+}$, only the fluorescence alteration intensity ratio of $\mathrm{Hg}^{2+}$ was very high. This meant the composite luminescence of assembly nanoparticles would change obviously only in the presence of $\mathrm{Hg}^{2+}$ (Fig. 8b). This high detection selectivity means the dual-emission composite nanoparticles could be used in some complex samples.

\section{Conclusions}

Dual-emission QDs/PCDs composite nanoparticles were prepared by a simple self-assembly method according to a hydrophobic-hydrophobic interaction. The dual-emission QDs/PCDs composite nanoparticles kept two different kinds of luminescence from PCDs and CdTe QDs. The luminescence color of the composite nanoparticles could be adjusted easily from blue to red simply by changing the CdTe QDs and the ratio between the amounts of PCDs and QDs. The composite nanoparticles possess high sensitivity and selectivity in detecting $\mathrm{Hg}^{2+}$; the detection limit of $\mathrm{Hg}^{2+}$ was $10 \mathrm{nM}$ by luminescence spectrum, and $100 \mathrm{nM}$ by visual observation. Because of the FRET in the composite nanoparticles, the luminescence color could be changed during the detecting of $\mathrm{Hg}^{2+}$; thus, it might be applied in some visible detecting areas.

\section{Conflicts of interest}

The authors declare no competing financial interest.

\section{Acknowledgements}

This work was supported by the NSFC (21404047), NSFC (51433003).

\section{References}

1 X. J. Cui, Y. H. Li, S. Bachmann, M. Scalone, A. E. Surkus, K. Junge, C. Topf and M. Beller, J. Am. Chem. Soc., 2015, 137, 10652-10658.

2 S. J. Zhu, Q. N. Meng, L. Wang, J. H. Zhang, Y. B. Song, H. Jin, K. Zhang, H. C. Sun, H. Y. Wang and B. Yang, Angew. Chem., Int. Ed., 2013, 52, 3953-3957.

3 H. J. Wang, C. H. Dong, P. Q. Zhao, S. Wang, Z. Y. Liu and J. Chang, Int. J. Pharm., 2014, 466, 307-313.

4 Y. F. Zhou, Q. Wang, B. Song, S. C. Wu, Y. Y. Su, H. M. Zhang and Y. He, Biomaterials, 2015, 72, 38-48.

5 D. Saika, P. Dutta, N. Sen Sarma and N. C. Adhikary, Sens. Actuators, B, 2016, 230, 149-156.

$6 \mathrm{~W}$. Wu, J. Shen, P. Banerjee and S. Q. Zhou, Biomaterials, 2010, 31, 8371-8381.

7 R. G. Chaudhuri and S. Paria, Chem. Rev., 2012, 112, 23732433.

8 C. S. Wu, M. K. K. Oo and X. D. Fan, ACS Nano, 2010, 4, 5897-5904.
9 Y. Ning, H. Zhang, J. S. Han, C. H. Yang, Y. Liu, D. Zhou and B. Yang, J. Mater. Chem., 2011, 21, 6837-6843.

10 Y. Fu, C. Q. Ding, A. W. Zhu, Z. F. Deng, Y. Tian and M. Jin, Anal. Chem., 2013, 85, 11936-11943.

11 H. Y. Chen, B. W. Li, M. Zhang, K. Sun, Y. R. Wang, K. R. Peng, M. D. Ao, Y. R. Guo and Y. Q. Gu, Nanoscale, 2014, 6, 12580-12590.

12 X. L. Dai, Z. X. Zhang, Y. Z. Jin, Y. Niu, H. J. Cao, X. Y. Liang, L. W. Chen, J. P. Wang and X. G. Peng, Nature, 2014, 515, 96-99.

13 X. H. Li, X. R. Yang, L. H. Yuwen, W. J. Yang, L. X. Weng, Z. G. Teng and L. H. Wang, Biomaterials, 2016, 96, 24-32.

14 Y. Y. Su, Y. He, H. T. Lu, L. M. Sai, Q. N. Li, W. X. Li, L. H. Wang, P. P. Shen, Q. Huang and C. H. Fan, Biomaterials, 2009, 30, 19-25.

15 D. K. Tiwari, T. Jin and J. Behari, Int. J. Nanomed., 2011, 6, 463-475.

16 S. N. Baker and G. A. Baker, Angew. Chem., Int. Ed., 2010, 49, 6726-6744.

17 A. D. Zhao, Z. W. Chen, C. Q. Zhao, N. Gao, J. S. Ren and X. G. Qu, Carbon, 2015, 85, 309-327.

18 W. B. Lu, X. Y. Qin, S. Liu, G. H. Chang, Y. W. Zhang, Y. L. Luo, A. M. Asiri, A. O. Al-Youbi and X. P. Sun, Anal. Chem., 2012, 84, 5351-5357.

19 Y. Zhang, P. P. Cui, F. Zhang, X. T. Feng, Y. L. Wang, Y. Z. Yang and X. G. Liu, Talanta, 2016, 152, 288-300.

20 Y. H. Wang, C. Zhang, X. C. Chen, B. Yang, L. Yang, C. L. Jiang and Z. P. Zhang, Nanoscale, 2016, 8, 5977-5984.

21 H. J. Yu, R. Shi, Y. F. Zhao, G. I. N. Waterhouse, L. Z. Wu, C. H. Tung and T. R. Zhang, Adv. Mater., 2016, 28, 9454-9477.

22 X. Y. Zhang, Y. Zhang, Y. Wang, S. Kalytchuk, S. V. Kershaw, Y. H. Wang, P. Wang, T. Q. Zhang, Y. Zhao, H. Z. Zhang, T. Cui, Y. D. Wang, J. Zhao, W. W. Yu and A. L. Rogach, ACS Nano, 2013, 7, 11234-11241.

23 J. P. Wang, L. Yang, B. H. Liu, H. H. Jiang, R. Y. Liu, J. W. Yang, G. M. Han, Q. S. Mei and Z. P. Zhang, Anal. Chem., 2014, 86, 3338-3345.

24 H. Zhang, Z. C. Cui, Y. Wang, K. Zhang, X. L. Ji, C. L. Lu, B. Yang and M. Y. Gao, Adv. Mater., 2003, 15, 777-780.

25 H. Ding, S. B. Yu, J. S. Wei and H. M. Xiong, ACS Nano, 2016, 10, 484-491.

26 L. L. Pan, S. Sun, A. D. Zhang, K. Jiang, L. Zhang, C. Q. Dong, Q. Huang, A. G. Wu and H. W. Lin, Adv. Mater., 2015, 27, 7782-7787.

27 H. Yu, R. Shi, Y. Zhao, G. I. N. Waterhouse, L. Wu, C. Tung and T. Zhang, Adv. Mater., 2016, 28, 9454-9477.

28 D. Yao, Y. Liu, W. Zhao, H. Wei, X. Luo, Z. Wu, C. Dong, H. Zhang and B. Yang, Nanoscale, 2013, 5, 9593.

29 Q. S. Zeng, Z. L. Chen, Y. Zhao, X. H. Du, F. Y. Liu, G. Jin, F. X. Dong, H. Zhang and B. Yang, ACS Appl. Mater. Interfaces, 2015, 7, 23223-23230.

30 Y. B. Lou, Y. X. Zhao, J. X. Chen and J. J. Zhu, J. Mater. Chem. C, 2014, 2, 595-613.

31 H. Z. Sun, H. Zhang, J. Ju, J. H. Zhang, G. Qian, C. L. Wang, B. Yang and Z. Y. Wang, Chem. Mater., 2008, 20, 6764-6769. 